\title{
Composite Higgs Boson in the Unified Subquark Model of All Fundamental Particles and Forces
}

\author{
Hidezumi Terazawa ${ }^{1,2}$, Masaki Yasuè ${ }^{3}$ \\ ${ }^{1}$ Center of Asia and Oceania for Science (CAOS), Higashi-kurume, Japan \\ ${ }^{2}$ Midlands Academy of Business \& Technology (MABT), Leicester, UK \\ ${ }^{3}$ Department of Physics, Tokai University, Hiratsuka, Japan \\ Email: terazawa@mri.biglobe.ne.jp, yasue@keyaki.cc.u-tokai.ac.jp
}

Received 19 January 2014; revised 15 February 2014; accepted 11 March 2014

Copyright (C 2014 by authors and Scientific Research Publishing Inc.

This work is licensed under the Creative Commons Attribution International License (CC BY).

http://creativecommons.org/licenses/by/4.0/

\section{(c) (i) Open Access}

\section{Abstract}

In the unified subquark model of all fundamental particles and forces, the mass of the Higgs boson in the standard model of electroweak interactions $\left(m_{H}\right)$ is predicted to be about $2 \sqrt{6} m_{W} / 3$ (where $m_{W}$ is the mass of the charged weak boson), which agrees well with the experimental values of 125 - $126 \mathrm{GeV}$ recently found by the ATLAS and CMS Collaborations at the LHC. It seems to indicate that the Higgs boson is a composite of the iso-doublet subquark-antisubquark pairs well described by the unified subquark model with either one of subquark masses vanishing or being very small compared to the other.

\section{Keywords}

\section{Composite Higgs Boson; Unified Subquark Model; Higgs Boson Mass; Subquark Masses}

What most of us could expect to find in high energy experiments at the Large Hadron Collider was the Higgs boson $(H)$, which was the only fundamental particle that had not been found in the standard model of electroweak interactions [1]-[3]. In the unified composite models of all fundamental particles and forces [4]-[6], where not only quarks and leptons but also gauge bosons as well as the Higgs boson are all composites of subquarks, the more fundamental particles in nature, the mass of the Higgs boson has been predicted in the following three ways:

In general, in composite models of the Nambu-Jona-Lasinio type [7], the Higgs boson appears as a composite 
state of fermion-antifermion pairs with the mass twice as much as the fermion mass. The unified subquark model of the Nambu-Jona-Lasinio type [8] [9] has predicted the following two sum rules:

$$
m_{W}=\left[3\left(m_{w_{1}}^{2}+m_{w_{2}}^{2}\right) / 2\right]^{1 / 2}
$$

and

$$
m_{H}=2\left[\left(m_{w_{1}}^{4}+m_{w_{2}}^{4}\right) /\left(m_{w_{1}}^{2}+m_{w_{2}}^{2}\right)\right]^{1 / 2},
$$

where $m_{w_{1}}$ and $m_{w_{2}}$ are the masses of the weak-iso-doublet spinor subquarks called wakems standing for weak and electromagnetic ( $w_{i}$ for $i=1,2$ ) while $m_{W}$ and $m_{H}$ are the masses of the charged weak boson $(W)$ and physical Higgs boson in the standard model, respectively. By combining these sum rules, the following relation has been obtained if the subquarks are iso-symmetric as $m_{w_{1}}=m_{w_{2}}$ :

$$
m_{w}: m_{W}: m_{H}=1: \sqrt{3}: 2 \text {. }
$$

From this relation, the wakem and Higgs boson masses have been predicted as

$$
m_{w}=m_{w} / \sqrt{3}=46.4 \mathrm{GeV}
$$

and

$$
m_{H}=2 m_{W} / \sqrt{3}=92.8 \mathrm{GeV}
$$

for $m_{W}=80.4 \mathrm{GeV}$ [10]. On the other hand, if $m_{w_{1}}=0$ or $m_{w_{2}}=0$, the other relation can be obtained:

$$
m_{w}: m_{W}: m_{H}=1: \sqrt{3 / 2}: 2 \text {. }
$$

From this relation, the non-vanishing wakem and Higgs boson masses can be predicted as

$$
m_{w}=m_{w} / \sqrt{3 / 2}=65.6 \mathrm{GeV}
$$

and

$$
m_{H}=2 m_{W} / \sqrt{3 / 2}=131 \mathrm{GeV}
$$

for $m_{W}=80.4 \mathrm{GeV}$ [10]. More generally, from the two sum rules, the Higgs boson mass can be bounded as

$$
92.8 \mathrm{GeV}=2 m_{W} / \sqrt{3} \leq m_{H} \leq 2 \sqrt{6} m_{W} / 3=131 \mathrm{GeV} .
$$

In the unified quark-lepton model of the Nambu-Jona-Lasinio type [8] [9], the following two sum rules for $m_{W}$ and $m_{H}$ have been predicted:

$$
m_{W}=\left(3\left\langle m_{q, l}^{2}\right\rangle\right)^{1 / 2}
$$

and

$$
m_{H}=2\left(\sum m_{q, l}^{4} / \sum m_{q, l}^{2}\right)^{1 / 2},
$$

where $m_{q, l}$ s are the quark and lepton masses and \langle\rangle denotes the average value for all the quarks and leptons. If there exist only three generations of quarks and leptons, these sum rules completely determine the top quark and Higgs boson masses [11] [12] as

$$
m_{t} \cong(2 \sqrt{6} / 3) m_{W}=131 \mathrm{GeV}
$$

and

$$
m_{H} \cong 2 m_{t} \cong(4 \sqrt{6} / 3) m_{W}=263 \mathrm{GeV} .
$$

Furthermore, triplicity of hadrons, quarks, and subquarks [13] tells us that these sum rules can be further extended to the approximate sum rules of

$$
m_{W} \cong\left(3\left\langle m_{B, l}^{2}\right\rangle\right)^{1 / 2}
$$


and

$$
m_{H} \cong 2\left(\sum m_{B, l}^{4} / \sum m_{B, l}^{2}\right)^{1 / 2},
$$

where $m_{B, l} \mathrm{~s}$ are the canonical baryon and lepton masses and \langle\rangle denotes the average value for all the canonical baryons and leptons. The canonical baryon means either one of $p, n$ and other ground-state baryons of spin 1/2 and weak-isospin 1/2 consisting of a quark heavier than the $u$ and $d$ quarks and a scalar and isoscalar diquark made of $u$ and $d$ quarks. If there exist only three generations of quarks and leptons, these sum rules completely determine the masses of the canonical topped baryon, $T$, and the Higgs boson as

$$
m_{T} \cong 2 m_{W}=161 \mathrm{GeV}
$$

and

$$
m_{H} \cong 2 m_{T} \cong 4 m_{W}=322 \mathrm{GeV} .
$$

Therefore, if the Higgs boson is found with the mass between $92.8 \mathrm{GeV}$ and $131 \mathrm{GeV}$, it looks like a composite state of subquark-antisubquark pairs. If it were found heavier with $m_{H}$ around $263 \mathrm{GeV}$ or even 322 $\mathrm{GeV}$, it could be taken as a bound state of $t \bar{t}$ (topponium) or $T \bar{T}$ (topped-baryonium), respectively. If it were found with the mass lying between these typical masses, it might be taken as a mixture of subquarkantisubquark pairs and quark-antiquark pairs, etc.

Very recently, the ATLAS and CMS Collaboration experiments at the CERN Large Hadron Collider have almost excluded the two ranges for the Higgs boson mass: the one lower than $114 \mathrm{GeV}$ and the other between $141 \mathrm{GeV}$ and $476 \mathrm{GeV}$ [14]-[23] [24]-[30], which disagrees with both the prediction in the unified quark-lepton model of the Nambu-Jona-Lasinio type [8] [9] and that in the unified baryon-lepton model of the Nambu-JonaLasinio type [13]. Instead, the prediction in the unified subquark model [8] [9] (92.8 GeV $\left.\leq m_{H} \leq 131 \mathrm{GeV}\right)$ shows a right ballpark on which the mass of the Higgs boson in the standard model should land. Moreover, the fact that the experimental values of $m_{H}=125-126 \mathrm{GeV}$ recently found by the ATLAS and CMS Collaborations are very close to the predicted one of $m_{H}=2 \sqrt{6} m_{W} / 3=131 \mathrm{GeV}$ seems to strongly suggest that either one of $m_{w_{1}}$ and $m_{w_{2}}$ vanishes or is much smaller than the other. In fact, if indeed $m_{H}=126 \mathrm{GeV}$ [14]-[23] [24]-[30], the two sum rules completely determine the subquark masses in the unified subquark model as $\left(m_{w_{1}}, m_{w_{2}}\right)=(13.3 \mathrm{GeV}, 64.3 \mathrm{GeV})$ or $(64.3 \mathrm{GeV}, 13.3 \mathrm{GeV})$ for $m_{w}=80.4 \mathrm{GeV}$ [10]. It seems to indicate that the Higgs boson is a composite of the iso-doublet spinor subquark-antisubquark pairs well described by the unified subquark model with either one of subquark masses vanishing or being very small compared to the other. We believe that this conclusion of the present paper is not only very important in high energy physics but also very intriguing in physics or in science in general. Let us hope that the future LHC (and also ILC) experiments will tell us whether the unified subquark model is a viable model of all fundamental particles and forces!

\section{Acknowledgements}

H.T. thanks Professor Keiichi Akama and Professor Yuichi Chikashige for very useful helps in correcting errors in the original manuscripts.

\section{References}

[1] Glashow, S.L. (1961) Nuclear Physics B, 22, 579.

[2] Salam, A. (1968) Weak and Electromagnetic Interactions. In: Svartholm, N., Ed., Elementary Particle Physics, Almqvist and Wiksell, Stockholm, 368.

[3] Weinberg, S. (1967) Physical Review Letters, 19, 1264.

[4] Terazawa, H. (1984) Subcomponent Models. In: Meyer, A. and Wieczorek, E., Eds. Proceedings of the 22nd International Conference on High Energy Physics, Leipzig, Akademie der Wissenschaften der DDR, Zeuthen, Vol. I, 63.

[5] Terazawa, H. (2003) Current Topics in Hadro-Particle Physics. In: Bogolyubov, P.N., Jenkovszky, L.L. and Magas, V.V., Eds., Proceedings of International Conference “New Trends in High-Energy Physics”, Alushta, Crimea, Bogolyubov Institute for Theoretical Physics, Kiev, Ukrainian Journal of Physics, 48, 1292.

[6] Terazawa, H. (2011) Masses of Fundamental Particles. In: Bogolyubov, P.N. and Jenkovszky, L.L. Eds., Proceedings of the XXII-d International Conference on New Trends in High-Energy Physics, Alushta (Crimea), Bogolyubov Institute for Theoretical Physics, Kiev, 352. 
[7] Nambu, Y. and Jona-Lasinio, G. (1961) Physical Review Letters, 122, 345. http://dx.doi.org/10.1103/PhysRev.122.345

[8] Terazawa, H., Chikashige, Y. and Akama, K. (1977) Physical Review D, 15, 480.

[9] Terazawa, H. (1980) Physical Review D, 22, 184.

[10] Beringer, J., et al. (Particle Data Group) (2012) Physical Review D, 86, Article ID: 010001. http://dx.doi.org/10.1103/PhysRevD.86.010001

[11] Terazawa, H. (1980) Physical Review D, 22, 2921.

[12] Terazawa, H. (1990) Physical Review D, 41, 3541 (E).

[13] Terazawa, H. (1990) Modern Physics Letters A, 5, 1031. http://dx.doi.org/10.1142/S0217732390001153

[14] ATLAS Collaboration (2011) European Physical Journal, 71, 1728.

[15] ATLAS Collaboration (2011) Physical Review Letters, 107, Article ID: 221802.

[16] ATLAS Collaboration (2011) Physics Letters B, 705, 435.

[17] ATLAS Collaboration (2012) Physical Review Letters, 108, Article ID: 111803.

[18] ATLAS Collaboration (2012) Physics Letters B, 710, 49.

[19] ATLAS Collaboration (2012) Physics Letters B, 710, 383.

[20] ATLAS Collaboration (2012) Physics Letters B, 716, 1.

[21] ATLAS Collaboration (2012) Physical Review D, 86, Article ID: 032003.

[22] ATLAS Collaboration (2013) Physics Letters B, 726, 88.

[23] ATLAS Collaboration (2013) Physics Letters B, 726, 120.

[24] CMS Colaboration (2012) Physics Letters B, 710, 26.

[25] CMS Colaboration (2012) Physics Letters B, 710, 91.

[26] CMS Colaboration (2012) Physics Letters B, 710, 403.

[27] CMS Colaboration (2012) Physics Letters B., 716, 30.

[28] CMS Colaboration (2012) Physical Review Letters, 109, Article ID: 121801.

[29] CMS Colaboration (2012) Journal of High Energy Physics, 09, 111.

[30] CMS Colaboration (2013) Journal of High Energy Physics, 06, 081. 